\title{
CRISIS RURAL, POBREZA Y HAMBRE EN CHIAPAS
}

\author{
Rural Crisis, Poverty and Hunger in Chiapas
}

\author{
Daniel Villafuerte-Solís
}

Resumen: La imagen descarnada de la crisis rural y la pobreza en el México de la segunda década del siglo XXI se expresa con toda claridad en las entidades tradicionalmente más pobres (Chiapas, Guerrero y Oaxaca), que en 2012 sumaron cerca de cuatro millones de personas con hambre. En Chiapas, aun cuando se reporta una reducción de 5.6 puntos porcentuales de la población que padece hambre, la situación adquiere rasgos de mayor complejidad toda vez que entre las causas del levantamiento armado del Ejército Zapatista de Liberación Nacional figuran la pobreza y el hambre. En este artículo se analizan, desde una perspectiva relacional, la crisis en el campo chiapaneco, la pobreza y el hambre, con el sustento de la teoría de la crisis y los datos duros generados por algunas dependencias federales.

Palabras clave: crisis rural, pobreza, dependencia alimentaria, hambre.

Abstract: The stark image of the rural crisis and poverty in Mexico in the second decade of the century is expressed clearly in the traditionally poorer states, Chiapas, Guerrero and Oaxaca, which ones in 2012 add up about four million hungry people. In Chiapas, although a reduction of 5.6 percentage points is reported in people who suffer from hunger. The situation acquires more complex features because two of the reasons behind the armed uprising of the Zapatista Army of National Liberation were the poverty and the hunger. In this article I analyze, the crisis in rural Chiapas, the poverty and the hunger, from a relational perspective, having as support the theory of crisis and the data generated by some federal agencies.

Keywords: rural crisis, poverty, food dependency, hunger.

Daniel Villafuerte Solís, doctor en ciencias sociales por la Universidad Autónoma Metropolitana, México. Profesor-investigador del Centro de Estudios Superiores de México y Centroamérica de la Universidad de Ciencias y Artes de Chiapas. Temas de especialización: migración, desarrollo, fronteras, seguridad y procesos de integración regional. Correo electrónico: gasoda_2000@yahoo.com.mx.

Enviado a dictamen: 22 de mayo de 2014. Aprobación: 17 de septiembre de 2014.

Revisiones: 1 . 


\section{Introducción}

$\mathrm{M}$ ás allá del carácter efectista que distingue al nuevo gobierno encabezado por el presidente Enrique Peña Nieto, la Cruzada Nacional contra el Hambre revela el fracaso del modelo neoliberal que había prometido empleo y prosperidad. La crisis del modelo se descubre de manera grotesca en los 53.3 millones de pobres — quinientos mil más de lo estimado en 2010 - y en los 11.5 millones de personas en pobreza extrema (CONEVAL, 2013). Más aún, se manifiesta de manera descarnada en el número de personas con problemas de acceso a la alimentación, que, según la misma fuente, alcanzó en 2012 la cifra de 27.4 millones de personas, el 23.3\% de la población del país.

Éste es el otro rostro del México de los tratados de libre comercio, del México en la OCDE. Chiapas es quizá el mejor ejemplo del fracaso del modelo de economía abierta y de los programas asistenciales que han intentado compensar los males del desarrollo neoliberal. Y, sin embargo, el gobierno actual insiste en sostener el mismo modelo. A este respecto son ilustrativas las declaraciones del secretario de Hacienda, Luis Videgaray, quien manifestó que “[...] sólo con la aprobación de más reformas estructurales, como la energética y hacendaria, México podrá generar crecimientos económicos mayores que permitan a millones de mexicanos salir de la pobreza [...] (Miranda, 2013). La historia se ha encargado de demostrar que el crecimiento económico por sí mismo no es garantía para superar la desigualdad y la pobreza en ningún país del mundo, y sin embargo se recurre a la retórica para evadir la responsabilidad del Estado.

Inmersos en este contexto, en la presente comunicación se analizan las causas que subyacen a la pobreza y el hambre en Chiapas, con el telón de fondo de la importancia de los programas sociales y los recursos financieros asignados para superar, en alguna medida, los problemas que han adquirido carácter estructural. Uno de los puntos de partida será entender la naturaleza de la crisis rural y de la producción agropecuaria, así como sus consecuencias. Se comienza por situar analíticamente la crisis, luego se aborda la crisis en el México rural, para bajar al análisis de Chiapas, y finalmente plantear algunas consideraciones conclusivas.

\section{La crisis y el México rural}

En general, la crisis se refiere a una variación en el estado, un cambio, una alteración. En economía hay por lo menos tres modelos de interpretación de la crisis: 1) se articula con los mecanismos monetarios, 2) está asociada a las innovaciones tecnológicas, o 3) se relaciona con el vínculo entre inversión y consumo. La crisis se refiere a un momento de quiebre en el funcionamiento de un sistema; es un cambio cualitativo.

La idea de crisis se vincula con el concepto de equilibrio, que puede ser estable - tiende a regresar a la misma posición - o inestable - tiempo para alcanzar un nuevo tipo de equilibrio después de la perturbación-. El momento en que se alcanza un nuevo equilibrio marca el fin de la crisis, pero no necesariamente su solución. Holloway (1990) define la crisis como una ruptura en el proceso normal de cambio. Es el momento crucial de una enfermedad, cuando la muerte o la recuperación están en juego. Cuando se aplica al desarrollo histórico y social, la crisis no se refiere únicamente a tiempos difíciles, sino a saltos cualitativos, de manera que el concepto de crisis es esencial en cualquier teoría del cambio social.

En México, desde finales de los años sesenta, comenzó a hablarse de crisis agrícola y rural. En 1980 se decía que "A medida que se ha profundizado la crisis rural, a partir del año de 1965, se ha hecho dada vez más vivo y ampliado el debate sobre el tema en la sociedad mexicana. Han participado en él todos los sectores, desde las más diversas posiciones ideológicas, y se observa intensidad semejante en las instituciones académicas y en los foros políticos" (Esteva, 1980: 185).

Durante la segunda mitad de los años seenta y en los ochenta, buena parte de la producción académica se centró en el debate en torno al campesinado y la agricultura tradicional. Esto se derivaba de la creencia de que la superación de la crisis sólo sería posible si se atendía al vasto sector del campesinado que producía alimentos. Se avanzó en el entendimiento sobre el campesinado 
mexicano y buena parte de la discusión estuvo centrada en los que Ernest Feder (1977) llamó "campesinistas" y "descampesinistas", estos últimos posicionados en la inevitabilidad de la extinción del campesinado y su conversión en proletarios. En el tratamiento de estos temas figuran diversos autores que se convirtieron en referentes obligados: Arturo Warman (1972), Roger Bartra et al. (1975), Gustavo Esteva (1980), Rodolfo Stavenhagen (1968), Blanca Rubio (1987), Armando Bartra (1985), Gustavo Gordillo (1988), José Luis Calva (1988) y Alejandro Schejtman (1982), entre muchos otros.

Hoy, en tiempos de neoliberalismo, la crisis adquiere nuevos rasgos que se expresan en la profundización de la pobreza estructural y en la nueva pobreza, la exclusión social, el hambre, las migraciones internas e internacionales, así como la instauración del neoextractivismo como un modo de acumulación que destruye de forma acelerada los recursos productivos, contamina el ambiente, genera violencia y pone en riesgo la vida de miles de seres humanos.

A partir de aquí emerge una discusión que ha venido ampliándose, especialmente en América del Sur, en torno al posdesarrollo y el buen vivir (Alimonada, 2012; Madoery, 2012; Esteva, 2009; Escobar, 2009; Gudynas, 2009). Este debate parte de una crítica radical a la concepción del desarrollo impuesta por la visión estadounidense poco después de la Segunda Guerra Mundial, cuya práctica termina cancelando las promesas de una vida mejor y se evidencia en el crecimiento de la pobreza, el hambre y el despojo.

\section{La crisis alimentaria en el marco del neoliberalismo}

No se puede ocultar que hoy estamos frente a nuevos problemas: no se trata sólo de una crisis de producción, de pérdida de autosuficiencia y de soberanía alimentaria, sino también de una crisis de los campesinos y de la sociedad rural — que se refleja en la pobreza, el hambre y la migración-, y de una crisis ecológica. Se trata de una crisis global que está poniendo en riesgo la supervivencia misma del planeta.

El Tratado de Libre Comercio de América del Norte (TLCAN) colocó a México bajo una peligrosa dependencia y le obligó a un cambio en el patrón de consumo alimentario que está generando mayor subordinación al mercado con efectos en la salud: somos el primer país en el mundo con problemas de obesidad infantil; la primera causa de muerte es la diabetes, ${ }^{1}$ en la ciudad y en el campo se consume cantidad de refrescos y productos procesados con escaso o nulo valor nutricional, y en muchos hogares rurales se ha sustituido el consumo de maíz por harina, lo que ha generado una dependencia del mercado y ha posicionado a las empresas productoras de harina, tales COMO MASECA, ${ }^{2}$ MINSA, AGROINSA y HARIMASA.

Como se puede observar en el cuadro 1 , el déficit en la balanza agropecuaria y agroindustrial creció en la última década en un 220\%; el déficit en la balanza de productos agropecuarios se incrementó en un $2111 \%$; los cinco productos básicos más importados registraron un incremento del orden del 219\%; y las importaciones de maíz, un $443.7 \%$.

Elcreciente déficit está relacionado conel incremento sostenido de los precios que han venido experimentando los productos agropecuarios en el mercado mundial, frente a lo cual la política gubernamental poco o nada ha hecho. Además, en 2011 la agricultura y la ganadería en los estados del norte del país sufrieron grandes pérdidas a causa de una de las sequías más devastadoras de los últimos setenta años, ${ }^{3}$ con más de dos millones de hectáreas dañadas y más de cien mil cabezas de ganado muertas por falta de alimento y de agua.

ElTLCAN dejó a miles de productores y consumidores en manos del mercado, que impone las condiciones de precios deteriorando la economía familiar. En este sentido, es importante observar cómo en la última década se han venido incrementando los precios internacionales de los alimentos (ver cuadro 2), lo que explica en buena medida el alarmante aumento en el número de personas con hambre en México, incluso en lugares donde antes eran superavitarios en producción de alimentos.

En correspondencia con el incremento de los precios de los alimentos en el mercado internacional, se observa un desmedido aumento en el valor de las importaciones: de acuerdo con las cifras del Banco de 
México, las importaciones de cereales, carnes y despojos comestibles se incrementaron en un 12.4\% entre 2009 y 2010, al pasar de 5746956000 dólares, a 6460500000 de dólares —en números redondos, de casi seis mil millones de dólares a 6.5 mil millones de dólares-. Esta última cifra es mayor que el gasto asignado a la Secretaría de Agricultura, Ganadería, Desarrollo Rural, Pesca y Alimentación (SAGARPA) para el año 20ll. En el año 2012, las importaciones de trigo, maíz, semilla de soya y carne sumaron poco más de diez mil millones de dólares (Banco de México, 2013).

El deterioro del campo ha sido brutal, al grado de provocar un éxodo masivo del campo a las ciudades y a Estados Unidos. A partir del año 2000, emigraron del país medio millón de personas en promedio al año, fenómeno que vino a engrosar el número de mexicanos que vive en Estados Unidos de forma clandestina, lo que llevó a la crisis al sistema migratorio del país del norte.

\section{Implicaciones de la crisis para Chiapas: pobreza y hambre}

¿Cómo ha repercutido la crisis en Chiapas? Chiapas constituye un ejemplo visible de las consecuencias de la crisis, un estado que arrastra un esquema productivo dominado por cultivos tradicionales, con una población de campesinos e indígenas muy significativa; una entidad que prácticamente concluyó su reforma agraria en el año 2000, con fuertes rezagos sociales y con casi el $75 \%$ de su población en condiciones de pobreza.

La crisis se refleja en la caída de la producción y en el valor de ésta, lo que se observa en la aportación que hace el sector agropecuario en la generación de riqueza en el conjunto de la economía chiapaneca: en 2009 no llegó al 10\% del PIB, indicador que contrasta notablemente con una población mayoritariamente rural. También lo podemos ver en la cantidad de población que trabaja en el campo, que en los últimos años ha venido disminuyendo; ahora hay más trabajadores en el comercio y en los servicios que en la agricultura. El $38 \%$ trabaja en el campo y el $46 \%$ se emplea en el sector terciario.
Una gran cantidad de campesinos ha emigrado para trabajar en Estados Unidos. En el VIII Censo agrícola, ganadero y forestal 2007 y en el IX Censo ejidal 2007 (INEGI, 2008) se registra un dato muy interesante: la mayoría de los jóvenes en quinientos ejidos y comunidades de Chiapas — poco más del 20\% del total — ha abandonado la familia: en 141, han emigrado hacia áreas urbanas del país; y en 282, hacia Estados Unidos. En el resto -77-, migraron hacia otras áreas rurales del país. Este fenómeno constata el impacto de la crisis en el campo chiapaneco, que se refleja en un proceso de "desruralización" y en el surgimiento de una "nueva ruralidad".

El proceso anterior ha venido acompañado por el crecimiento de la pobreza y de la nueva pobreza, sobre todo en el campo. Según los datos más recientes del CONEVAL (2013), que corresponden al año 2012, Chiapas sigue siendo el estado con el mayor número de pobres de todas las entidades federativas del país: cerca del $75 \%$ de su población vive en condiciones de pobreza. Supera a Oaxaca en casi 13 puntos porcentuales, a Puebla en 9.2 y a Guerrero en 5. Chiapas tiene alrededor de 1.3 millones más de pobres que Guerrero y Oaxaca. Los cinco estados del sur presentados en el cuadro 4 cuentan con el 31.2\% de los pobres del país y con el 50.6\% de los pobres extremos.

En pobreza extrema, Chiapas también ocupa el primer lugar nacional dado que el $32.2 \%$ de su población se encuentra en esta condición - tres veces más que el promedio nacional-; supera en casi nueve puntos porcentuales a Oaxaca. La pobreza extrema alcanza a poco más de 1.6 millones de personas, lo que coloca a este estado en una situación de altísima vulnerabilidad social, susceptible a la inestabilidad y la protesta, así como a la conformación de movimientos sociales. Estos son los promedios estatales, pero la situación en los municipios rurales y con población mayoritariamente indígena es todavía más grave.

Sólo para mencionar algunos ejemplos, puede afirmarse que los municipios con mayor número de pobres son San Juan Cancuc y Aldama, con el 97.3\% de su población en esta condición. A estos le sigue muy de cerca un grupo de municipios con más del 96\% de su 
población en condiciones de pobreza: Chalchihuitán, Sitalá, San Andrés Larráinzar y Santiago El Pinar.

Entre los municipios con mayor número de pobres extremos se encuentran Mitontic, Chenalhó, Larráinzar, Pantelhó y Sitalá, donde más del 70\% de su población se encuentra en estas condiciones. Además, se registra un grupo de tres municipios que están en una situación extremadamente crítica pues alcanzan casi el 80\%: Aldama, Chalchihuitán y San Juan Cancuc.

Pero esto no es todo, hay otro grupo compuesto por 31 municipios que tiene un alto porcentaje de su población en condiciones de pobreza, entre los que vale la pena destacar a Ocosingo, Huitiupán, San Andrés Duraznal, Nicolás Ruiz, Amatán, Pantepec, San Lucas, Teopisca e Ixhuatán, donde más del 90\% de su población está en condiciones de pobreza y casi el 50\% en pobreza extrema.

La pobreza material de la mayoría de los chiapanecos es de tal magnitud, sobre todo en comunidades indígenas y campesinas, que la Cruzada Nacional contra el Hambre ha incluido a 55 de los 118 municipios que integran el estado. En esta lista se encuentran aquellos que hace dos décadas eran líderes en la producción de granos básicos. Nos referimos a Chiapa de Corzo, Chicomuselo, Frontera Comalapa, Tapachula, Villa Corzo y Villaflores. En estos se encuentran los nuevos pobres, que emergieron con la aplicación de las políticas de ajuste que implicaron la disminución de subsidios y créditos blandos a los campesinos.

De los 55 municipios incluidos en la Cruzada, 25 presentan una situación de extrema vulnerabilidad (ver cuadro 5). Estos municipios abarcan una población de 786535 habitantes, el 16.3\% de la población total, cuyo nivel de pobreza general oscila en un rango del $90.2 \%$ al $97.3 \%$, de pobreza extrema entre el $54.3 \%$ y el $80.5 \%$, y una población con hambre que va del $31 \%$ al $60 \%$.

De estos veinticinco municipios, siete aportan el mayor número de habitantes con hambre, cuya suma alcanza la cifra de 180 431. Estos municipios son San Juan Cancuc, Chenalhó, Chilón, Zinacantán, Chamula, Oxchuc y Pueblo Nuevo Solistahuacán. Chilón aporta el mayor número de personas con hambre, con una cifra que asciende a 56180 personas. Este municipio se caracteriza porque la mayoría de sus habitantes son indígenas, con una población predominante joven: el $46.2 \%$ de la población de 3 años y más se incluye en el rango comprendido entre los 5 y 19 años. En cuanto a la población que habla lengua indígena, ésta representa el 96.4\% de la población mayor de 3 años; y de ésta, el $45.5 \%$ no habla español. Estas cifras ponen de relieve el estado de emergencia de estos municipios olvidados, que son el blanco de un discurso gubernamental que plantea hasta lo "imposible" su propósito de sacarlos de la exclusión. Como ejemplo reciente está el programa de los veintiocho municipios de menor índice de desarrollo humano implementado por el gobierno de Juan Sabines, alineado a los Objetivos de Desarrollo del Milenio, que no logró cambiar la realidad y que, sin embargo, recibió el premio Más Naciones Unidas en México. En esa ocasión, Sabines expresó lo siguiente:

Hoy se focaliza la tercera parte del presupuesto del estado, 10 veces más de lo que se invirtió el sexenio anterior, eso significa dar sacrificios posiblemente hasta compromisos de campaña, hay que dejar a un lado carreteras para atender los ODM de manera concreta, por eso se focaliza la inversión en los 28 municipios posibles de Chiapas y de los más pobres de México (El Orbe, 25 de octubre de 2010).

Otro de los proyectos ligados al combate a la pobreza y la marginación fue el programa Ciudades Rurales Sustentables. En su Sexto informe de gobierno, Sabines Guerrero refirió que las

[...] ciudades rurales sustentables es uno de los proyectos más importantes de esta administración, que convirtió a Chiapas en pionero a nivel nacional, así como en ejemplo para la réplica de la estrategia en otras entidades. Es considerada como una de las mejores prácticas gubernamentales que se puso en marcha en la entidad para combatir la dispersión y erradicar la pobreza extrema de maneraefectiva, a través de la distribución territorial de la población a las potencialidades de desarrollo regional, concentración de la población que habita en localidades rurales dispersas y en zonas de riesgo, 
dotación de servicios e instrumentación de proyectos productivos con empleos mejor remunerados (Gobierno del Estado de Chiapas, 2012: 69, cursivas nuestras).

El gobierno de Sabines compró la idea según la cual una de las causas de la pobreza y la marginación era la dispersión de la población en muchas localidades —más de veinte mil-, y esto justificó sumas millonarias para la construcción de "ciudades rurales sustentables". Independientemente del equívoco de considerar la dispersión de la población como causante de la pobreza, se necesitaría construir entre 100 y 170 "ciudades rurales" para concentrar a los casi 2.5 millones de habitantes que están en alrededor de veinte mil localidades menores de dos mil quinientos habitantes, lo que sería una verdadera locura. En la administración de Sabines sólo pudieron concluirse dos - Nuevo Juan del Grijalva y Santiago El Pinar-, y dos más quedaron inconclusas: la de Ixhuatán, con 379 viviendas, y la Ciudad del Café en Jaltenango La Paz, con 625 casas y una inversión de poco más de 210 millones de pesos — 336000 pesos por cada casa- A pesar de haber sido el proyecto más importante del gobierno de Sabines, como explícitamente se indicó en su informe de gobierno, ha recibido fuertes críticas de sectores representativos de la sociedad, no sólo por la concepción misma del proyecto, que no tomó en cuenta las características específicas de la vivienda en el medio rural - cocinar con leña, tener cerca la parcela y animales de traspatio junto a la vivienda, etcétera-, sino también por el elevado costo de cada "ciudad rural" frente a su efectividad para mejorar las condiciones de vida de la gente. ${ }^{4}$

Por otra parte, durante los gobiernos del Partido Acción Nacional el gasto federal del principal programa de combate a la pobreza, Oportunidades, mantuvo un crecimiento sostenido, situación que está en contradicción con el discurso de reducción de la pobreza. Como puede verse en el cuadro 6, el gasto entre 2000 y 2011 se incrementó en más de un 500\% en el país y en Chiapas, lo que sugiere un crecimiento del número de hogares en pobreza. Esto se refleja en los datos del CONEVAL tanto en el ámbito nacional, como en el estatal.

Chiapas ocupa un lugar importanteen el presupuesto asignado al combate a la pobreza, cuya proporción del gasto nacional fue de un promedio muy cercano al 10\% durante el período 2000-2011. De hecho, la entidad es la segunda en importancia, después de Veracruz, por el monto de los recursos otorgados por Oportunidades. El tercer lugar lo ocupa Puebla, sigue en cuarto lugar el Estado de México, Oaxaca en quinto y Guerrero en sexto lugar. Los seis estados concentraron en 2011 el 49.73\% del total de recursos, con 29402.9 millones de pesos, lo que una vez más constata que el sur de México es el territorio donde se concentra la mayor cantidad de pobres y excluidos del país, y sin embargo, paradójicamente, es donde existen vastas riquezas en recursos naturales.

El crecimiento del gasto del programa Oportunidades en el ámbito nacional y en Chiapas guarda correspondencia con el aumento en el número de familias atendidas, que durante el periodo 2000-2011 se duplicó. Lo importante a destacar es que la proporción de familias atendidas con respecto al total registró una variación significativa en Chiapas con respecto al promedio nacional. Así, mientras que en el país se atendió el 22.2\% del total de familias, en el año 2011, en el caso de Chiapas se atendió el 62.4\%, lo que a todas luces refleja un crecimiento de la pobreza con relación a la población total.

En suma, Chiapas recibió entre 2000 y 2011 la cantidad de 38787 millones de pesos, equiparable al presupuesto de egresos de la entidad en 2008, que ascendió a 40382 millones de pesos. ¿Cómo se explica que a mayor presupuesto, mayor pobreza? Y es que el presupuesto de la entidad en los últimos siete años casi se duplicó al crecer un $88 \%$ y, sin embargo, los indicadores sociales no han avanzado en concordancia con el aumento presupuestal.

Los mayores incrementos en el gasto público ocurrieron entre 2008 y 2010. Al tomar como base el presupuesto de 2007, se puede ver que en 2010 éste había aumentado en un 56.3\%. En 2012, el último año de la administración de Sabines, ocurrió otro incremento 
significativo, que fue del 7.5\%. En el primer año de la nueva administración encabezada por Manuel Velasco Coello, el presupuesto aumentó en un $7.4 \%$. Con ello, Chiapas tiene el presupuesto más alto de su historia, con casi 67000 millones de pesos y, sin embargo, de manera contradictoria, el gobierno emitió un decreto de austeridad que sólo puede entenderse en el marco del endeudamiento que dejó la administración de Sabines, el cual, según la Secretaría de Hacienda y Crédito Público (SHCP), hasta diciembre de 2012, sumaba 16412.9 millones de pesos (SHCP, 2012). Al 31 de marzo de 2014, en un año y tres meses, la SHCP reportó un incremento de la deuda del orden del $26.6 \%$, por lo que la suma ascendió a 20773.6 millones de pesos (SHCP, 2014).

\section{Cambio en el patrón de cultivos como indicador de la crisis}

La crisis también se puede observar en los cambios en el patrón de cultivos: se impulsó un modelo de producción basado en la competitividad, de modo que nuevos productos pudieran ser colocados en los mercados. Ya no importa la autosuficiencia, como en otros tiempos, sino que se trata - se dice- de garantizar mayores ingresos a los campesinos para que compren sus alimentos en el mercado. En esta lógica, se inducen proyectos de reconversión productiva, que se basan en la sustitución de áreas dedicadas a la producción de alimentos básicos para el fomento de cultivos comerciales, en particular para la elaboración de agrocombustibles: piñón y palma africana. ${ }^{5}$ En este contexto, el gobierno de Juan Sabines, fiel al mandato del Proyecto Mesoamérica, creó un aparato burocrático para responder a estos propósitos: el Instituto de Reconversión Productiva y Bioenergéticos.

Producto de la política de reconversión productiva que ya se venía perfilando antes del gobierno de Sabines, se observa que entre los años 2000 y 2010 la superficie cosechada de maíz sufrió una disminución de 260393 hectáreas y la producción cayó en casi 493000 toneladas. En el mismo período, la de frijol disminuyó en 14401 hectáreas y el volumen cosechado experimentó una caída de casi siete mil toneladas. Por el contrario, la superficie cosechada de palma africana se incrementó en un $624 \%$, al pasar de 2748 hectáreas a 19 902, lo que impactó notablemente en la producción, que se incrementó en un 562\%. En números precisos, significó pasar de 51345 a 342037 toneladas. ${ }^{6}$

A tono con la política nacional, durante la administración del gobierno de Juan Sabines los esfuerzos se canalizaron hacia el impulso de productos destinados al mercado. Con la idea de la reconversión y diversificación productiva, se apoyaron proyectos que demandaba el mercado nacional, sin importar el mercado regional y el consumo de la mayoría de la población. En su quinto informe de gobierno, Sabines refería que

\begin{abstract}
Nuestra apuesta por la reconversión y diversificación productiva está basada en la reingeniería de los recursos naturales para establecer plantaciones perennes seleccionadas por el potencial de mercado, su adaptabilidad local, la rentabilidad para el productor y la capacidad para generar valor agregado a través de sistemas tecnológicos integrados territorialmente para combinar plantaciones con agricultura protegida, que diversifique fuentes de ingreso y genere flujos intensivos y complementarios (Gobierno del Estado de Chiapas, 2011: 233).
\end{abstract}

El gobierno de Sabines reiteró una y otra vez que su administración se distinguía por ser el "sexenio del campo", pero lo cierto es que el discurso no se reflejó en el presupuesto. Los datos del anexo II de su Sexto informe de gobierno revelan que durante el período 2007 2012 se destinaron poco más de 6259 millones de pesos al sector agropecuario y forestal, lo que representó únicamente el 1.9\% del presupuesto de ese etapa, que ascendió a casi 331000 millones de pesos. Para tener una idea del reducido gasto en el sector primario basta con compararlo con el gasto en transporte, que alcanzó 21000 millones de pesos, equivalente al 6.3\% del total; o con el gasto destinado a la coordinación de la política del gobierno, que ascendió a 6565 millones de pesos, casi el 2\% del total, o con el gasto del pago de la deuda, que fue de poco más de 5958 millones de pesos. Otra forma 
de medir la magnitud del gasto es con relación al PIB del estado, que únicamente representó el 0.5\%, sin incluir el correspondiente a 2012. ${ }^{7}$ Se trata de una cifra muy baja si tomamos en cuenta la importancia estratégica del campo en términos sociales y de estabilidad política pues, a pesar de la larga crisis que padece, el sector primario todavía emplea alrededor del 40\% de la población económicamente activa.

La administración de Sabines se caracterizó por el impulso a proyectos que nada tuvieron que ver con la autosuficiencia o la soberanía alimentaria. En la lógica de su gobierno, dejó que los recursos del Procampo y los del Programa Maíz Solidario ayudaran a reproducir el círculo de pobreza de los campesinos. Los campesinos han dejado de ser "hijos predilectos del régimen" (Warman, 1972), y ahora deambulan por las ciudades para cobrar en los bancos o en los cajeros automáticos los magros subsidios del Procampo. Otra parte de ellos ya se encuentra en los campos agrícolas de Estados Unidos o en la industria de la construcción.

En contrapartida, a los productores volcados en el mercado, que están en una posición mejor en cuanto a dotación de recursos productivos, la política gubernamental procuró brindarles apoyos; en particular destaca el programa de mecanización en áreas de mejores rendimientos. Evidentemente esto marca una diferencia radical con los campesinos que viven en los veinticinco municipios que hoy padecen hambre.

El programa de "tractorización" es una paradoja, pues lejos de procurar el empleo para que la gente del campo no emigre a las ciudades del interior o a Estados Unidos, con la mecanización se alienta la movilidad humana, además de que se descuidan las áreas campesinas más pobres que necesitan apoyos para producir sus propios alimentos y no depender del mercado, o depender en escala mínima.

Por otra parte, la superficie destinada al cultivo de café ha ido en aumento: en 1980 se registró una superficie de 142000 hectáreas; y en 2004, veinticuatro años después, la frontera cafetalera se había ampliado 242000 hectáreas. Esto significa que en veinticuatro años, a pesar de la crisis de los noventa, la superficie aumentó 100000 hectáreas; ya para 2010 la superficie sumaba 253541 hectáreas; es decir, aumentó en casi 12000 hectáreas en sólo dos años. La cada vez mayor dependencia del mercado ha provocado que una parte importante de campesinos y productores minifundistas reduzca o suprima las áreas dedicadas al cultivo de granos básicos para ampliar la superficie cultivada de café, que en tiempos de bajos precios, como los que ahora se están viviendo, genera una contracción del ingreso y, por consiguiente, los productores reducen su consumo de alimentos, que ahora tienen que comprar a precios cada vez más elevados.

Otros cultivos que también aumentaron su superficie cosechada fueron la caña de azúcar, en más de 4000 hectáreas; el mango, en casi 5000 hectáreas; y la palma africana, en casi 8000 hectáreas, aunque la superficie sembrada, según datos oficiales, ya alcanza las 100000 hectáreas. El reino del mercado y del dinero, traducido en política económica de producir para el mercado y descuidar la producción para el autoconsumo, ha implicado altos costos que se reflejan en los niveles de pobreza y hambre en amplios sectores de la población.

Junto con la disminución de la frontera maicera como consecuencia de las políticas neoliberales, y en particular de la denominada reconversión productiva, lo que más llama la atención es el cambio en la geografía del cultivo de maíz: la región Frailesca, emblemática, considerada en los años ochenta del siglo XX como el granero de Chiapas, ha sido reemplazada por otros municipios que antes se dedicaban a la ganadería bovina, entre los que figuran Ocosingo y Palenque, que juntos sumaron una superficie cosechada de casi 87000 hectáreas, frente a Villaflores y Villa Corzo, donde se cultivaron alrededor de 33000 hectáreas. Sin embargo, la producción difiere en cada uno de los grupos de municipios: mientras el primer grupo produjo 84740 toneladas, el segundo grupo, en menos de la mitad de la superficie del primero, generó una producción de 135835 toneladas. La diferencia estriba en el nivel de rendimientos, pues en Ocosingo y Palenque fue de 1.2 toneladas por hectárea, en tanto que en Villaflores y Villa Corzo se obtuvieron 4.35 toneladas. El cambio en el régimen de subsidios desincentivó la producción en áreas altamente productivas, como fue el caso de 
la región Frailesca, y desplazó la producción hacia regiones marginales de producción campesina.

Como compensación a la caída en la superficie y la producción de maíz, a partir de 2008 se implementó el programa denominado Maíz Solidario, ${ }^{8}$ que consistió en un "subsidio" a los productores de subsistencia. En 2008, el programa benefició a 240048 productores, con un monto de 250 millones de pesos; en el 2009 únicamente se apoyó a 210000 productores, con 210 millones de pesos; y en el 2010 a 200000 productores, con recursos que sumaron 180 millones de pesos. En este último año, el número de productores que se dejaron de apoyar fue de 40000 con relación al primer año de operación del programa, y la cantidad de recursos disminuyó en 70 millones de pesos. Se trata de un apoyo simbólico de entre 800 y 1000 pesos por campesino. En este contexto, el titular de Diconsa anunció a fines del año 2011 que para abastecer el consumo de maíz en Chiapas en los primeros meses de 2012 "se han importado de Sudáfrica 45000 toneladas". Es la paradoja en un estado que en otro tiempo exportó maíz a los estados vecinos.

Tres fenómenos sintetizan la crisis en Chiapas: la pobreza, el hambre y la migración, en especial la migración a Estados Unidos, que comenzó desde el año 1990 en las zonas cafetaleras afectadas por la disminución internacional de precios. En 2007, las remesas enviadas por los mexicanos llegaron a 25000 millones y las de los chiapanecos rebasaron los 800 millones; en 2010, por efecto de la crisis, bajaron a 574 millones de dólares, una disminución de 226 millones; sin embargo, esta disminuida cantidad representa diez veces más de lo que entregaba el gobierno del estado a través del programa Maíz Solidario. Las remesas siguen bajando: en el 2012 llegaron a Chiapas 572.7 millones de dólares, y la información para 2013 reporta una caída de casi un 6.6\% con relación al año anterior, al sumar 535 millones de dólares, casi 38 millones menos (Banco de México, 2014).

\section{Reflexiones finales}

Chiapas vive una prolongada y profunda crisis expresada en la magnitud de la pobreza, que adquiere mayores dimensiones en el medio rural y entre la población indígena. A la pobreza secular se ha sumado en las últimas dos décadas la nueva pobreza generada por las políticas de carácter neoliberal. La destrucción y el deterioro de los medios de vida, fundamentalmente de la tierra, los bosques y el agua, han contribuido a la situación de pobreza de miles de familias campesinas e indígenas, que hoy sobreviven principalmente de los programas asistencialistas que otorgan los gobiernos federal y estatal. En el año 2013, en el marco del programa Cruzada contra el Hambre, los recursos financieros estimados para Chiapas fueron de casi 10820 millones de pesos, prácticamente la mitad de los cuales se destinó al rubro de salud, seguido del Programa para Adultos Mayores y del programa productivo Procampo. Estos tres ejes concentraron el 68\% del presupuesto. El ámbito productivo es el que menor atención recibe, por lo que es difícil imaginar que una entidad donde todavía existe una enorme población que se dedica a cultivar la tierra pueda superar los rezagos productivos y mejorar la alimentación de su población. El principal objetivo de la Cruzada, que consistía en alcanzar "Cero hambre a partir de una alimentación y nutrición adecuada de las personas en pobreza multidimensional extrema y carencias de acceso a la alimentación", ${ }^{9}$ resulta una utopía o una retórica discursiva al observarse los escasos recursos destinados a la producción campesina.

La estructura del gasto social para Chiapas sigue la lógica definida para el país: en el presupuesto de egresos de la federación 2013, anexo 9, referido a "erogaciones para el desarrollo integral de los pueblos y comunidades indígenas" (DOF, 2012), aparece un gasto superior a los 74000 millones de pesos, distribuidos en 78 programas. Sin embargo, ocho de ellos concentran poco más del 65\% del presupuesto referido -48300 millones de pesos-; de éstos, el programa Oportunidades en sus tres versiones -Oportunidades-SEP, OportunidadesSEDESOL y Oportunidades IMSS - concentra el 24\% del presupuesto - 17 700 millones de pesos-; dos - Seguro Popular y Pensión para adultos mayoresreúnen el 17\%; dos programas de infraestructura -básica y económica de carreteras alimentadoras y caminos rurales - suman el 21.5\% — 15900 millones de pesos-; y uno - Procampo productivo- 
únicamente representa el 2.7\% de los recursos —-2000 millones de pesos- La misma historia se repite en el presupuesto de egresos 2014. En este presupuesto, del monto asignado al mismo rubro y anexo referido, que ascendió a poco más de 77000 millones de pesos (DOF, 2013b), 37642 millones de pesos, cantidad equivalente al 48.8\%, correspondieron al programa Oportunidades en sus distintas versiones, a pensión para adultos mayores y a seguro popular. La parte destinada al fomento productivo volvía a ser mínima con relación al gasto para consumo y servicios.

La deuda social histórica no podrá superarse con la Cruzada Nacional contra el Hambre, que recicla muchos de los programas que han demostrado poca eficacia. Las cifras del CONEVAL son contundentes en este sentido. En el medio rural radican muchas de las claves para mitigar la pobreza, la exclusión social, la migración y la violencia. Los jóvenes del campo se encuentran sin esperanzas, por lo que es muy frecuente que opten por abandonar sus lugares de origen para escapar de la miseria en la que están sumidas sus familias. Superar la crisis de sentido de grandes contingentes del campo resulta fundamental si se quiere ir más allá de un programa limitado a evitar el desbordamiento social. Parafraseando a Polanyi (2006), La gran transformación para superar la miseria y el hambre de millones de mexicanos, y de chiapanecos en particular, debe partir de reconocer la situación de crisis que vive el campo y la realidad económica y social de los grupos más vulnerables, como los jóvenes, los indígenas y las mujeres.

\section{Notas}

${ }^{1}$ Se estima que actualmente hay en el país más de diez millones de personas con diabetes, en su mayoría tipo 2. La obesidad en niños de 5 a 11 años aumentó un 124\% de 1999 a 2006.

2 "La industria de la harina de maíz está sumamente concentrada; sólo cuatro empresas dominan el mercado, de las que el Grupo Industrial Maseca es la número uno con $71 \%$ de participación de mercado, mientras MINSA,
AGROINSA y HARIMASA se dividen el resto (29\%)", Servicio de Información Agroalimentaria y Pesquera (SIAP). Disponible en http://www.campomexicano. gob.mx/portal_sispro/index.php?portal=maiz [consultado el 29 de septiembre de 2014].

${ }^{3}$ Un boletín publicado por el INEGI (2012) refiere que "Las cifras de la Secretaría de Agricultura, Ganadería, Desarrollo Rural, Pesca y Alimentación (SAGARPA) revelan que en el año agrícola del 2011 sumaron 2.7 millones las hectáreas siniestradas en siete de los principales cultivos, sobre todo en Sinaloa, Zacatecas y Guanajuato".

${ }^{4}$ Un cálculo estimado por vivienda, a partir de la información proporcionada por la gente que habita la "ciudad rural" Nuevo Juan del Grijalva, llega a la escandalosa cifra de cinco millones de pesos (Libert, 2012).

${ }^{5}$ En cuatro años, informó el gobierno de Juan Sabines, se establecieron 82282 hectáreas de plantaciones a través del programa de reconversión productiva, distribuidas de la siguiente manera: 28500 de frutales, 32935 de palma de aceite, 10766 de hule, 10000 de piñón y 81 de agricultura protegida. Como se puede ver, más de la mitad de la superficie reconvertida se dedicó a cultivos para la producción de aceite. Habrá que hacer una revisión más fina de las cifras, pues según el director del Instituto de Reconversión Productiva y Bioenergéticos, en 2011 se completaron 68000 hectáreas de palma africana, lo que significa que entre 2010 y 2011 se duplicó la superficie.

${ }^{6}$ Los datos fueron tomados del portal OEIDRUS Chiapas. Disponible en http://www.oeidrus-chiapas. gob.mx/ [consultado el 30 de junio de 2014].

${ }^{7}$ De acuerdo con la información del Sistema de Cuentas Nacionales del INEGI, la suma del producto interno bruto de Chiapas durante el período 2007-201l alcanzó la cifra de 1112031.7 millones de pesos, a precios corrientes (INEGI, s.f.).

${ }^{8}$ Véasehttp://www.secretariadelcampo.gob.mx/ subsecretarias/Agricultura/index.php.

${ }^{9}$ Artículo $2^{\circ}$ del decreto por el que se establece el Sistema Nacional para la Cruzada contra el Hambre (DOF, 2013a). 


\section{Referencias bibliográficas}

Alimonada, Héctor (2012), "Desarrollo, posdesarrollo y buen vivir: reflexiones a partir de la experiencia ecuatoriana”. En Crítica y Emancipación, Revista Latinoamericana de Ciencias Sociales, año IV, núm. 7, primer semestre. Argentina, pp. 27-58.

Banco de México (2012), Balanza de pagos. CE49. Importaciones totales. México. México, Banco de México. Disponible en http://www.banxico.org.mx/ SieInternet/consultarDirectorioInternetAction.do?ac cion=consultarCuadro\& $\&$ idCuadro=CE49\&sector $=1 \&$ locale $=e s$ [consultado el 2 de julio de 2012].

Banco de México (2014), Estadísticas. Balanza depagos. México, Banco de México. Disponible en http://www.banxico. org.mx/SieInternet/consultarDirectorioInternetAction. do?accion=consultarDirectorioCuadros\&sector=l\&se ctorDescripcion=Balanza [consultado el 10 de abril de 2014].

Bartra, Armando (1985), Losherederos de Zapata. Movimientos campesinos posrevolucionarios en México. México, Era.

Bartra, Roger et al. (1975), Caciquismo y poder político en el México rural. México, Instituto de Investigaciones Sociales-UNAM/Siglo XXI.

Calva, José Luis (1988), Los campesinos y su devenir en las economías de mercado. México, Siglo XXI.

Calva, José Luis (1993), La disputa por la tierra. La reforma al artículo 27 y la nueva Ley Agraria. México, Editorial Fontamara.

Camacho, Fernando (2013), "En Valle de Chalco, 58 por ciento de los habitantes vive en situación de miseria, México". En La Jornada, 26 de enero de 2013.

Cardoso, Fernando H. y Enzo Falleto (1977), Dependenciay desarrollo en América Latina. México, Siglo XXI.

CONEVAL (2011), Pobrezaen México yen las entidadesfederativas 2008-2010. México, CONEVAL. Disponible en http:// www.coneval.gob.mx/Informes/Interactivo/Medicion pobreza_2010.pdf [consultado el 23 de mayo de 2013].

CONEVAL (2013), Medición de la pobreza. Resumen ejecutivo. México, CONEVAL. Disponible en http://web.coneval.gob.mx/Medicion/Paginas/ Medici\%C3\%B3n/Pobreza\%202012/Resumen_ ejecutivo.aspx [consultado el 22 de mayo de 2014].
DOF (Diario Oficial de la Federal) (2012), Presupuesto de egresos de la federación para el ejercicio fiscal 2013. México, Secretaría de Gobernación, 27 de diciembre de 2012.

DOF (Diario Oficial de la Federación) (2013a), Decreto por el que se establece el Sistema Nacional para la Cruzada contra el Hambre. México, Secretaría de Gobernación, 22 de enero de 2013.

DOF (Diario Oficial de la Federal) (2013b), Presupuesto de egresos de la federación para el ejercicio fiscal 2014. México, Secretaría de Gobernación, 3 de diciembre de 2013.

El Orbe (2010), "Reconocen a Chiapas por objetivos del milenio". Tapachula, Chiapas, 25 de octubre de 2010.

Escobar, Arturo (2009), "Un minga para el postdesarrollo". En América Latina en Movimiento, núm. 445, año XXXIII, II época. Quito, Agencia Latinoamericana de Información, pp. 26-30.

Esteva, Gustavo (1980), La batalla en el México rural. México, Siglo XXI.

Esteva, Gustavo (2009), "Más allá del desarrollo". En América Latina en Movimiento, núm. 445, año XXXIII, II época. Quito, Agencia Latinoamericana de Información, pp. 1-5.

Feder, Ernest (1977), Campesinistas y descampesinistas. Tres enfoques divergentes (no incompatibles) sobre la destrucción del campesinado. México, Siglo XXI.

FAO (2014), "Índice de la FAO para los precios de los alimentos". S.l., FAO. Disponible en http://www. fao.org/worldfoodsituation/foodpricesindex/es/ [consultado el 8 de abril de 2014].

Gobierno del Estado de Chiapas (2011), Quinto informe de gobierno. Tuxtla Gutiérrez, Chiapas, Gobierno del Estado.

Gobierno del Estado de Chiapas (2012), Sexto informe de gobierno. Tuxtla Gutiérrez, Chiapas, Gobierno del Estado.

Gobierno de los Estados Unidos Mexicanos (2012), Anexo estadístico del sexto informe de gobierno. México, Presidencia de la República.

Gordillo, Gustavo (1988), Los campesinos al asalto del cielo. De la explotación estatal a la apropiación campesina. México, Siglo XXI.

Gudynas, Eduardo (2009), "El día después del desarrollo". En América Latina en Movimiento, núm. 445, año 
XXXIII, II época. Quito, Agencia Latinoamericana de Información, pp. 31-33.

Holloway, John (1990), "Crisis, fetichismo y composición de clase”. En Relaciones, núm. 3. México, Universidad Autónoma Metropolitana, pp. 1-30.

INEGI (2008), VIII Censo Agrícola, Ganadero y Forestal 2007 y IXCenso Ejidal 2007. Aguascalientes, INEGI.

INEGI (2012), "Estadísticas a propósito del día mundial de la lucha contra la desertificación y la sequía". Aguascalientes, INEGI. Disponible en http://www. inegi.org.mx/inegi/contenidos/espanol/prensa/ Contenidos/estadisticas/2013/sequia0.pdf [consultado el 28 de junio de 2013].

INEGI (2013), Boletín de estadísticas vitales 2009. Aguascalientes, INEGI.

INEGI (s.f.), "Banco de información económica. Cuentas nacionales. Producto interno bruto por entidad federativa". Aguascalientes, INEGI. Disponible en http://www.inegi.org.mx/sistemas/bie/ [consultado el 10 de diciembre de 2013].

Libert, Antoine (2012), Dialógicas del territorio en Chiapas: un análisis sistémico-complejo del Proyecto Mesoamérica. Tesis de maestría en desarrollo rural regional. San Cristóbal de Las Casas, Chiapas, Universidad Autónoma Chapingo.

Madoery, Oscar (2012), "El desarrollo como categoría política”. EnCríticay Emancipación.RevistaLatinoamericana de Ciencias Sociales, año IV, núm. 7, primer semestre. Argentina, pp. 59-83.

Miranda, Juan Carlos (2013), "Programas asistenciales no han resuelto la pobreza”. En La Jornada, 30 de julio de 2013.

Pereda, Cristina F. (2013), "40,000 agentes vigilarán frontera entre México y Estados Unidos”. En El País, 26 de junio de 2013.

Polanyi, Karl (2006[1957]), La gran transformación. Los orígenes políticos y económicos de nuestro tiempo. México, Fondo de Cultura Económica.
Rubio, Blanca (1987), Resistencia campesina y explotación rural en México. México, Ediciones Era.

SAGARPA (2014), "Balanza disponibilidad-consumo". México, SAGARPA. Disponible en http://www. siap.gob.mx/balanza-disponibilidad-consumo/ [consultado el 4 de abril de 2014].

Salazar, Francisco y Olga Rodríguez (2011), "México sufre su peor sequía en 70 años". En Infobae América, 4 de diciembre de 2011. Disponible en http://america.infobae. com/notas/39406-Mexico-sufre-su-peor-sequia-en-70anos [consultado el 8 de diciembre de 2012].

Schejtman, Alejandro (1982), Economía campesino y agricultura empresarial. Tipología de productores del agro mexicano. México, CEPAL/Siglo XXI.

SHCP (Secretaría de Hacienda y Crédito Público) (2012), "Obligaciones financieras de entidades federativas y municipios por tipo de deudor. 2012. Cuarto trimestre". México, SHCP. Disponible en http://www.hacienda.gob.mx/ESTADOS/DEUDA_ PUBLICA_EFM/2012/Paginas/4toTrimestre.aspx [consultado el 30 de enero de 2014].

SHCP (Secretaría de Hacienda y Crédito Público) (2014), "Obligaciones Financieras de Entidades Federativas y Municipios por tipo de deudor. 2014. Primer trimestre". México, SHCP. Disponible en http:// www.hacienda.gob.mx/Estados/Deuda_Publica EFM/2014/Paginas/lerTrimestre.aspx [consultado el 28 de mayo de 2014].

SIAP (Sistema de Información Agroalimentaria y Pesquera) (s.f.), "Maíz”. México, SIAP. Disponible en http://www.campomexicano.gob.mx/portal_sispro/ index.php?portal=maiz [consultado el 10 de diciembre de 2013].

Stavenhagen, Rodolfo et al. (1968), Neolatifundismo y explotación. México, Nuestro Tiempo.

Warman, Arturo (1972), Los campesinos, hijos predilectos del régimen, México, Nuestro Tiempo. 
Cuadro 1. Balanza agropecuaria y agroindustrial, importación de cinco productos y maíz 2000-2011 (en miles de dólares)

\begin{tabular}{|c|c|c|c|}
\hline Concepto/ año & 2000 & 2011 & 2013 \\
\hline Balanza agropecuaria y agroindustrial & -1583619 & -5067342 & -2953060 \\
\hline Balanza de productos agropecuarios & -128020 & -2831527 & -1025262 \\
\hline Importación de cinco productos* & 2237305 & 7137833 & 6269720 \\
\hline Importaciones de maíz & 822 & 2989321 & 2053003 \\
\hline
\end{tabular}

*Trigo, maíz, arroz, sorgo y soya.

Fuente: elaboración propia con base en datos del Banco de México (2014).

Cuadro 2. Índice de la FAO para los precios de los alimentos

\begin{tabular}{|c|c|c|c|c|c|c|}
\hline Año & $\begin{array}{c}\text { Índice de los precios } \\
\text { de los alimentos }\end{array}$ & Carne & $\begin{array}{l}\text { Productos } \\
\text { lácteos }\end{array}$ & Cereales & $\begin{array}{l}\text { Aceites y } \\
\text { grasas }\end{array}$ & Azúcar \\
\hline 2000 & 91 & 96 & 95 & 86 & 68 & 116 \\
\hline 2001 & 95 & 100 & 105 & 87 & 67 & 123 \\
\hline 2002 & 90 & 90 & 81 & 94 & 87 & 98 \\
\hline 2003 & 98 & 96 & 95 & 99 & 101 & 101 \\
\hline 2004 & 113 & 114 & 123 & 107 & 112 & 102 \\
\hline 2005 & 118 & 124 & 135 & 101 & 103 & 140 \\
\hline 2006 & 127 & 121 & 130 & 119 & 113 & 210 \\
\hline 2007 & 161 & 131 & 219 & 163 & 172 & 143 \\
\hline 2008 & 201 & 161 & 223 & 232 & 227 & 182 \\
\hline 2009 & 160 & 141 & 149 & 170 & 153 & 257 \\
\hline 2010 & 188 & 158 & 207 & 179 & 197 & 302 \\
\hline 2011 & 229 & 183 & 229 & 241 & 254 & 369 \\
\hline 2012 & 213 & 182 & 194 & 236 & 224 & 306 \\
\hline 2013 & 210 & 184 & 243 & 219 & 193 & 251 \\
\hline
\end{tabular}

Fuente: FAO. Disponible en http://www.fao.org/worldfoodsituation/wfs-home/foodpricesindex/es/ 
Cuadro 3. Importaciones de maíz amarillo y blanco (miles de toneladas)

\begin{tabular}{||c||r||r||r||}
\hline \multicolumn{1}{|c|}{ Año } & Maíz amarillo & Maíz blanco & \multicolumn{2}{c|}{ Total } \\
\hline \hline 2009 & 7045 & 163 & 7208 \\
\hline \hline 2010 & 7276 & 506 & 7782 \\
\hline 2011 & 7777 & 1432 & 9209 \\
\hline 2012 & 8100 & 1344 & 9444 \\
\hline 2013 & 7020 & 512 & 7532 \\
\hline
\end{tabular}

Fuente: SAGARPA (2014).

Cuadro 4. Estados más pobres del país, 2012. Porcentaje de la población en pobreza y número de personas

\begin{tabular}{||l||r||r||r||r||}
\hline \multicolumn{1}{|c||}{ Entidad } & Pobreza (porcentaje) & Miles de personas & $\begin{array}{c}\text { Pobreza extrema } \\
\text { (porcentaje) }\end{array}$ & Miles de personas \\
\hline \hline Chiapas & 74.7 & 3782.3 & 32.2 & 31.7 \\
\hline \hline Guerrero & 69.7 & 2442.9 & 1629.2 \\
\hline \hline Puebla & 64.5 & 3878.1 & 11.6 & 1059.1 \\
\hline \hline Oaxaca & 61.9 & 2434.6 & 23.3 & 916.6 \\
\hline \hline Veracruz & 52.6 & 4141.8 & 14.3 & 1122.0 \\
\hline
\end{tabular}

Fuente: CONEVAL (2013). 
Cuadro 5. Municipios con mayor número de pobres y en situación de pobreza alimentaria

\begin{tabular}{|c|c|c|c|c|}
\hline Municipio & $\begin{array}{c}\text { Población } \\
\text { Total }\end{array}$ & Pobreza general & Extrema pobreza & $\begin{array}{c}\text { Pobreza } \\
\text { alimentaria }\end{array}$ \\
\hline San Juan Cancuc & 32538 & 97.3 & 80.5 & 50.9 \\
\hline Aldama & 5033 & 97.3 & 78.8 & 40.5 \\
\hline Chalchihuitán & 14378 & 96.8 & 79.8 & 47.0 \\
\hline Santiago El Pinar & 3233 & 96.5 & 69.2 & 35.9 \\
\hline San Andrés Larráinzar & 21961 & 96.3 & 73.0 & 47.6 \\
\hline Chanal & 13158 & 96.1 & 69.1 & 68.0 \\
\hline Pantelhó & 23258 & 96.0 & 70.3 & 38.6 \\
\hline Mitontic & 13370 & 95.9 & 71.8 & 55.5 \\
\hline Maravilla Tenejapa & 11660 & 95.8 & 68.2 & 45.7 \\
\hline Tenejapa & 47390 & 95.7 & 65.2 & 36.9 \\
\hline Chenalhó & 38891 & 95.6 & 72.3 & 46.8 \\
\hline Sitalá & 12785 & 95.6 & 74.6 & 39.1 \\
\hline Chilón & 124017 & 95.3 & 70.6 & 45.3 \\
\hline Amatenango del Valle & 8038 & 94.9 & 67.6 & 53.4 \\
\hline El Bosque & 21107 & 94.9 & 63.5 & 36.7 \\
\hline Zinacantán & 43476 & 94.9 & 64.8 & 52.8 \\
\hline Chamula & 83373 & 94.8 & 69.7 & 31.0 \\
\hline Ocotepec & 12603 & 94.7 & 63.1 & 38.3 \\
\hline Sabanilla & 31789 & 94.4 & 66.3 & 42.3 \\
\hline Tila & 86780 & 94.2 & 69.3 & 33.4 \\
\hline Oxchuc & 49819 & 93.5 & 62.0 & 52.7 \\
\hline San Lucas & 8038 & 93.4 & 50.0 & 44.4 \\
\hline Jitotol & 20800 & 93.3 & 64.6 & 37.3 \\
\hline Huixtán & 24829 & 92.9 & 60.5 & 44.6 \\
\hline Pueblo N. Solistahuacán & 34,201 & 90.2 & 54.3 & 42.2 \\
\hline Total & 786525 & & & \\
\hline
\end{tabular}

Fuente: CONEVAL (2011). 
Cuadro 6. Gasto federal en el Programa de Desarrollo Humano Oportunidades (millones de pesos corrientes)

\begin{tabular}{|l||r|r||r|}
\hline \multicolumn{1}{|c|}{ Año } & \multicolumn{1}{c|}{ Total nacional } & \multicolumn{1}{c|}{ Chiapas } & \multicolumn{1}{c|}{ Porcentaje } \\
\hline \hline 2000 & 9586.9 & 960.5 & 10.01 \\
\hline 2001 & 12393.8 & 1293.5 & 10.43 \\
\hline 2002 & 17003.8 & 1094.9 & 6.43 \\
\hline 2003 & 22331.1 & 2326.1 & 10.41 \\
\hline 2004 & 25651.7 & 2553.8 & 9.95 \\
\hline 2005 & 29954.2 & 2906.1 & 9.70 \\
\hline 2006 & 33525.7 & 3215.8 & 9.59 \\
\hline 2007 & 36769.2 & 3667.0 & 9.97 \\
\hline \hline 2008 & 41706.5 & 4287.4 & 10.27 \\
\hline 2009 & 46698.8 & 4781.9 & 10.23 \\
\hline 2010 & 57348.9 & 5719.2 & 9.97 \\
\hline 2011 & 59119.2 & 5990.8 & 10.13 \\
\hline
\end{tabular}

Fuente: Gobierno de los Estados Unidos Mexicanos (2012). 